\title{
Partial chromosome Y deletion
}

INSERM

\section{Source}

INSERM. (1999). Orphanet: an online rare disease and orphan drug data base. Partial chromosome Y deletion. ORPHA:1646

Male sterility due to chromosome $Y$ deletion is characterized by a severe deficiency of spermatogenesis. Chromosome $Y$ deletions are a frequent genetic cause of male infertility. 